\title{
Scar formation in the vestibular sensory epithelium after aminoglycoside toxicity
}

\author{
Lawrence Z. Meiteles ', Yehoash Raphael * \\ Department of Otolaryngology-Head and Neck Surgeny and Kresge Hearing Research Institute, University of Michigan Medical Center, \\ 1301 East Ann Street, Ann Arbor, MI 48109-0506, USA
}

(Received 4 October 1993; Revision received 4 May 1994; Accepted 15 May 1994)

\begin{abstract}
Hair cell degeneration and the repair process due to differing types of trauma have been studied extensively in the organ of Corti. It has been determined that, during scar formation, after differing types of trauma to the auditory sensory system, the reticular lamina is maintained with adherens junctions and tight junctions. We investigated the repair process within the vestibular epithelium. Hair cell degeneration was induced by the unilateral application of streptomycin to the inner ears of guinea pigs. Whole mount preparations of all five vestibular organs were processed and examined by fluorescence, light and electron microscopy. Scar formation was seen as early as 4 days post-treatment with streptomycin and was noted to coincide with hair cell degeneration. Neighboring supporting cells swelled and filled the space beneath the degenerating hair cell. Between three and five supporting cells participate in the reparative process. The distribution of cytokeratin is also altered during scar formation. The area once occupied by the hair cell becomes filled with cytokeratin-rich processes of supporting cells. It appears that differing numbers of supporting cells are involved in the reparative process within the vestibular sensory epithelium as compared to the auditory system. The reticular lamina remains intact at all times. This may possibly prevent mixing of fluids between different compartments in the inner ear and dysfunction of the vestibular sensory organs.
\end{abstract}

Key words: Vestibular epithelium; Aminoglycosides; Mammal; Degeneration; Regeneration; Hair cells

\section{Introduction}

The aminoglycoside antibiotics were introduced into clinical use in 1944, when streptomycin was the first antibiotic discovered to be effective against tuberculosis (Hinshaw and Feldman, 1945; Schuknecht, 1974; Fee, 1980). Use of the drug revealed that parenteral administration of 2-3 g daily resulted in loss of vestibular function within 2 to 4 weeks. With higher daily doses or prolonged treatment, hearing loss also occurred. Streptomycin's side effect of vestibulotoxicity was quickly put into use for the treatment of vertigo

\footnotetext{
"Presented, in part, at the Midwinter Meeting, Association for Research in Otolaryngology, St. Petersburg Beach, FL, February 1993.

* Corresponding author. Fax: (313) 764-0014.

${ }^{1}$ Dr. Meiteles is currently at New York Medical College, Department of Otolaryngology, Westchester Medical Center, Macy Pavilion Room 1042-B, Valhalla, New York 10595, USA.
}

(Fowler, 1948). When used systemically, bilateral vestibular loss occurs, and hence, it is only used today for the treatment of bilateral Meniere's disease (Langman et al., 1992). Intramuscular injections of 1-2 $\mathrm{g}$ are given daily until vestibular symptoms resolve or until hearing loss occurs. This treatment protocol is based upon the antibiotic's selectivity in destroying vestibular function prior to cochlear function. The use of streptomycin for the treatment of tuberculosis has also increased in the past few years due to the virulent strains seen in AIDS patients. There has also been a renewed interest clinically in the unilateral transtympanic application of aminoglycoside antibiotics for vestibular function ablation (Nedzelski et al., 1993).

Hair cell (HC) degeneration due to aminoglycoside toxicity has been studied extensively in the organ of Corti (Hawkins, 1976). A regulated mechanism of scar formation takes place in order to maintain the functional integrity of the organ of Corti after $\mathrm{HC}$ degeneration (Raphael and Altschuler, 1991b). Supporting cells 
(SCs) invade the subapical space of degenerating hair cells prior to complete loss of the $\mathrm{HC}$ and while the reticular lamina is still intact. Hence, scar formation coincides with hair cell loss, and the integrity of the reticular lamina is maintained.

The effect of streptomycin on the vestibular epithelium of guinea pigs has been investigated at the ultrastructural level (Duvall and Wersall, 1963). The first sign of damage observed was disintegration of the internal membranes of mitochondria in the supranuclear portion of the cell and swelling of mitochondria. Stereocilia were noted to swell and sometimes fuse to form giant cilia. The stereocilia and kinocilium were also seen to disappear, and the supranuclear portion of the cell would bulge out into the endolymphatic space. Some cells were completely pushed out into the lumen of the endolymphatic space, whereas others lost their surface membranes with all stereocilia and part of the cytoplasm. SCs were reported to be normal. The authors postulated that the site of action of streptomycin was on the surface membrane, causing increased permeability and leading to the changes just described.

The effect of streptomycin on the vestibular epithelium of guinea pigs has been investigated at the cellular level (Lindeman, 1969). Streptomycin was injected into the middle ear space and its effect investigated 2-8 days later. The first change seen was the formation of pyknotic nuclei with dispersed chromatin. This was followed by clumping of stereocilia and cytoplasmic protrusion from the HCs into the luminal space. Lindeman also observed 'collapse figures' which he compared to phalangeal scars in the organ of Corti. He also reported that, after a few weeks, the SCs surrounding the degenerated hair cells filled the gap formed by loss of the HCs and the collapse figures disappeared. Selective sensitivity of the different vestibular organs to aminoglycoside toxicity was also quantified. HCs of the crista ampullaris were more sensitive to streptomycin toxicity than were those of the utricle. 'The saccule was most resistant to streptomycin toxicity. Type I HCs were more sensitive than type $11 \mathrm{HCs}$, and $\mathrm{HCs}$ in the central portion of the crista ampullaris were more sensitive than those in the periphery.

Small doses of streptomycin in cats were noted to produce only vestibular effects, whereas higher doses also produced high-frequency hearing loss (McGee and Olszewski, 1962). Ataxia developed on day 12 after daily intramuscular injections of streptomycin. The ataxia improved over the following 2-3 weeks. Animals were sacrificed 1 year after treatment. Histologic evaluation revealed approximately $50-90 \%$ loss of HCs without changes in the SCs or first-order neurons in the crista ampullaris. The utricle and saccule had only $10 \%$ loss of HCs. Vestibular nerve histopathology did not reveal effects attributable to streptomycin.
Scar formation due to varying types of trauma has been well documented in the auditory sensory epithelium (Bohne, 1976; Hawkins and Johnson, 1981; Raphael and Altschuler, 1991a,b). The early stages of $\mathrm{HC}$ degeneration have been delineated for the vestibular epithelium (Duvall and Wersall, 1963; Lindeman, 1969). Little information is available on scar formation in the vestibular epithelium. Recently, the guinea pig vestibular epithelium was shown to have a limited regenerative capability (Forge et al., 1993; Warchol et al., 1993). Herein we report our results on scar formation and the repair process within the vestibular sensory epithelium after aminoglycoside toxicity.

\section{Methods}

\section{Induction of hair cell loss and tissue harvesting}

Eleven pigmented guinea pigs weighing $250-400 \mathrm{~g}$ were used. Animals were anesthetized with a combination of Rompun $10 \mathrm{mg} / \mathrm{kg}$ and ketamine $40 \mathrm{mg} / \mathrm{kg}$. Under sterile conditions, a postauricular incision was made and the round window exposed. A 24-gauge angiocath needle attached to a tuberculin syringe was used to inject streptomycin solution directly into the inner ear. A total of $0.1 \mathrm{ml}$ was infused into the guinca pig's inner ear via the round window over a period of 3 s. Concentrations of $5-20 \mathrm{mg} / \mathrm{ml}$ of streptomycin (dissolved in normal saline) were used. An additional 0.1 $\mathrm{ml}$ was placed into the mastoid bulla overlying the oval and round windows. The surgical incision was then closed in layers. A sham operation was performed on the opposite ear to act as a control. The same steps were performed and artificial perilymph solution injected into the control inner ear. On occasion, a different concentration of streptomycin was used in the control ear.

Table 1 presents the number of animals in each group according to post-trauma recovery time, preparation method and dosage of drugs applied.

Animals were anesthetized with an intraperitoneal injection of $10 \mathrm{mg} / \mathrm{kg}$ Beuthanasia-D with subsequent cardiac perfusion of a $4 \%$ solution of paraformaldehyde in $0.1 \mathrm{M}$ phosphate buffer, $\mathrm{pH} 7.4$. The temporal

Table 1

Recovery time \# of animals Dosage Preparation technique

\begin{tabular}{llrl}
\hline 1 day & 1 & $20 \mathrm{ml}$ & fluorescence \\
4 days & 1 & $10 \mathrm{ml}$ & fluorescence \\
7 days & 2 & $5 \mathrm{ml}$ & fluorescence \\
7 days & 1 & $20 \mathrm{ml}$ & LM/EM \\
7 days & 1 & $10 \mathrm{ml}$ & $\mathrm{LM} / \mathrm{EM}$ \\
10 days & 1 & $10 \mathrm{ml}$ & LM/EM \\
10 days & 2 & $5 \mathrm{ml}$ & fluorescence \\
30 days & 1 & $5 \mathrm{ml}$ & fluorescence \\
90 days & 1 & $10 \mathrm{ml}$ & fluorescence \\
\hline
\end{tabular}


bones were then harvested, the bulla opened, and the stapes removed. Inner ear perfusion with fixative was then performed. After fixation, the osseous otic capsule was removed piecemeal by dissection. The vestibular sensory epithelia was harvested as whole tissue surface preparation with the aid of a dissecting microscope. Cochleae from these animals were processed simultaneously as control tissue, since the distribution of cytokeratins in the cochlea has been previously described. The cristae ampullaris of each canal (lateral, superior and posterior), and the utricle and saccule were studied.

\section{Vestibular function after unilateral vestibulotoxicity}

The clinical effects of the unilateral application of aminoglycosides were documented by close observation of the treated animals immediately upon awakening from anesthesia and hourly thereafter for $5 \mathrm{~h}$. Animals were examined for nystagmus, posturing and ambulating ability. Animals were examined 3 times daily until signs of vestibular dysfunction resolved.
Whole mount fluorescence histochemistry and microscopy

Eight animals were processed for fluorescence histochemistry. The tissue was permeabilized with $0.3 \%$ Triton-X-100 for $10 \mathrm{~min}$. This permeabilization regimen is routinely used in our laboratory to prepare inner ear epithelia for cytochemistry. It consistently allows intracellular localization of different cytoplasmic proteins. Specimens were then incubated with $4 \%$ normal goat serum for $30 \mathrm{~min}$ to block nonspecific antibody binding. After rinsing with PBS, the tissue was incubated for $1.5 \mathrm{~h}$ with monoclonal pan-anti-cytokeratin antibody (Boerhinger Mannheim, Indianapolis, IN), which binds to an epitope common to all cytokeratins. Specimens were then washed and incubated with rhodamine conjugated goat anti-mouse IgG for $30 \mathrm{~min}$. FITC conjugated phalloidin was used at a 1:75 dilution in combination with the secondary antibody to double label for actin. The tissue was then washed again and mounted on a welled microscope slide in glycerol-PBS solution.

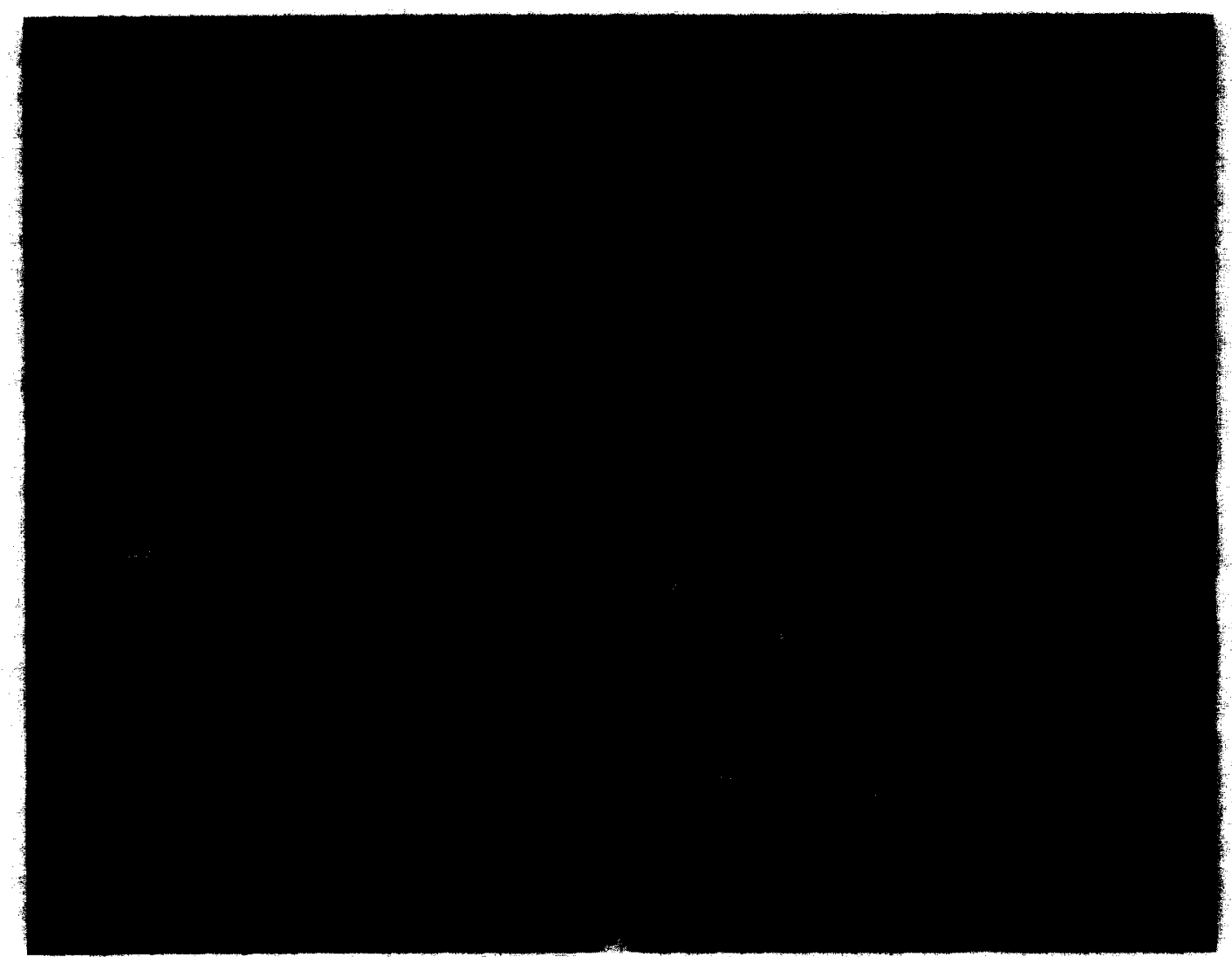

Fig. 1. Whole mount of the normal utricle labeled with fluorescent phalloidin. The left side of the micrograph is focused at the level of the cuticular plate and the right side at the stereocilia. Actin-specific label is found in the stereocilia (black arrows), cuticular plate, and in association with the adherens junction forming a circumferential ring around the apical surface of hair cells and supporting cells. The apical surfaces of hair cells (h) are round and easily distinguishable from supporting cells. Supporting cell apical surfaces are polygonal in shape and contain an actin-free zone in the central portion (white arrow). Groups of four to six supporting cells surround each hair cell. Bar, $10 \mu \mathrm{m}$. 
Specimens were examined, as whole mounts, with a Leitz Orthoplan microscope equipped for epifluorescence and phase contrast and a confocal microscope. The confocal microscope was a Biorad MRC-600 attached to an inverted microscope (Nikon Diaphot) with Nomarski optics capability. Images were obtained using X60 (NA $=1.4)$ and $\mathrm{X} 100(\mathrm{NA}=1.4)$ oil objectives. Selected images were produced with a hardware zoom. Photomicrographs of specimens were taken using Kodak T-max films.

\section{Tissue sections: light and electron microscopy}

Three animals each were processed for light (LM) and electron microscopy (EM). Specimens were obtained as whole tissue preparations, as described above for fluorescence histochemistry; however, the immunoperoxidase technique was used to label cytokeratins. Specimens were permeabilized with $0.3 \%$ Triton$\mathrm{X}-100$, rinsed, and incubated with $5 \%$ normal horse serum to block nonspecific antibody binding. Specimens were then incubated with the anti-cytokeratin antibody, rinsed with PBS, and incubated with the second antibody for $1.5 \mathrm{~h}$. The second antibody used was biotinylated and affinity purified. The biotinylated secondary antibody was then detected using a preformed macromolecular complex between avidin and the biotinylated enzyme, employing the Vectastain $\mathrm{ABC}$ Kit from Vector Laboratories (Burlingame, CA). In control specimens, labeling was as described above, except for omitting the anti-cytokeratin antibody.

The specimens were postfixed in $1 \%$ osmium tetroxide, dehydrated in alcohol, embedded in Epon 812 and sectioned with a glass knife or diamond knife (for LM and EM, respectively) on a LKB Ultramicrotome. Semi-thin sections for LM were stained with toludine blue, examined and photographed with a Leitz Dialux microscope. Thin sections of $90 \mathrm{~nm}$ were stained with

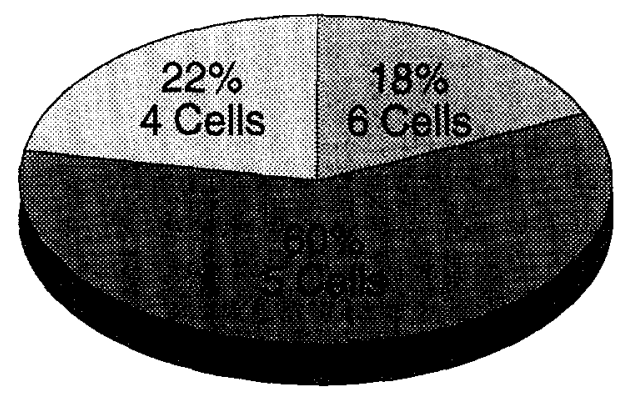

4 Cells $=22 \%$

5 Cells $=60 \%$

6 Cells $=18 \%$

Fig. 2. The number of supporting cells surrounding each hair cell was counted in normal cristae. Between four and six supporting cells surround each individual hair cell. The majority of hair cells were surrounded by five supporting cells $(N=108)$.
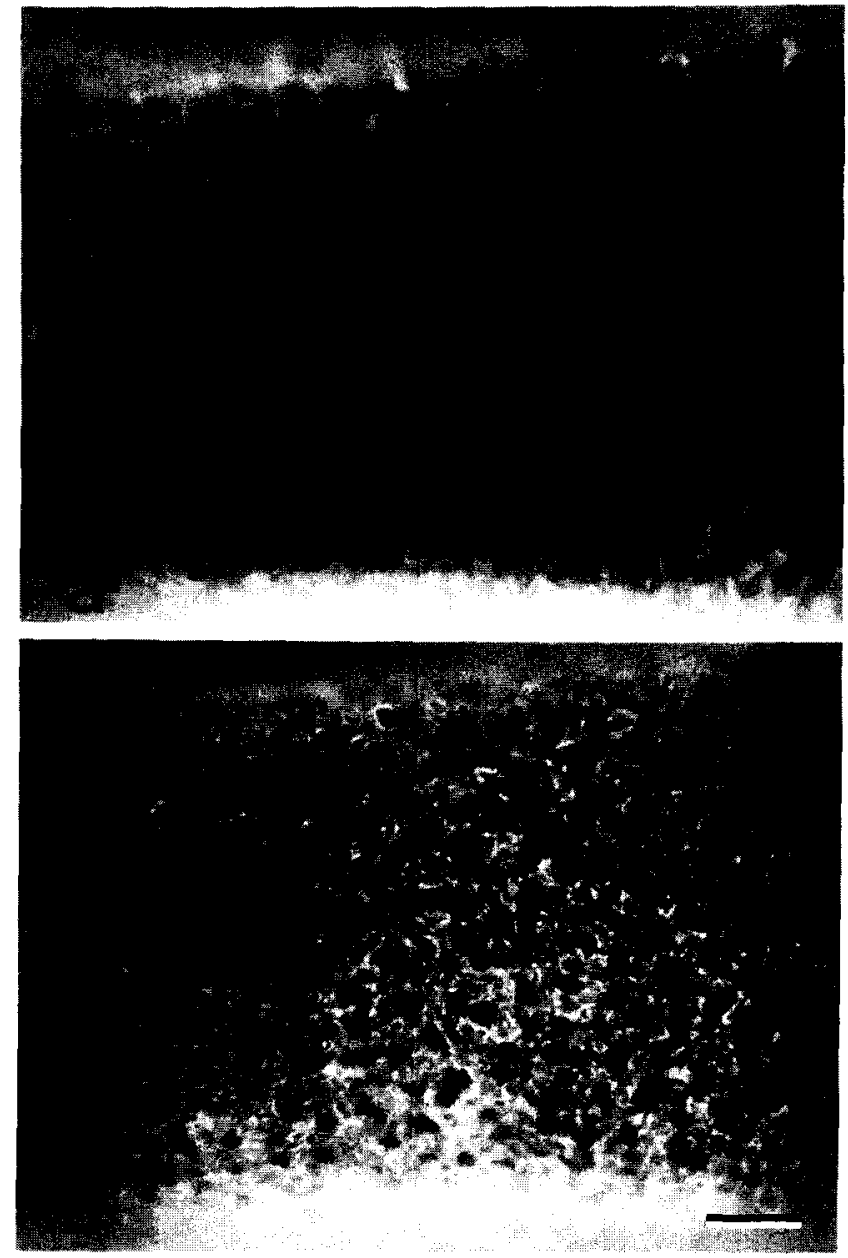

Fig. 3. A whole mount of a crista ampullaris double-labeled for actin (a) and cytokeratins (b). The entire surface of the crista could be observed, and the photographed field shows the midportion, focused at the level of the reticular lamina. (a) The distribution of actin in the stereocilia and cuticular plate delineated the normal organization of cells in this epithelium. (b) Cytokeratin staining was restricted to supporting cells. The areas occupied by hair cells are not stained, appearing as dark circles. Bar. $20 \mu \mathrm{m}$.

uranyl acetate and lead citrate and examined with a JEOL 1200 EX electron microscope.

\section{Results}

Immunofluorescence microscopy of the vestibular sensory epithelium reveals a unique pattern of actin and cytokeratin staining, similar to the auditory system. Actin-specific staining of vestibular HCs is seen within the stereocilia, cuticular plate, and forming a circumferential ring of adherens junctions at their apical surface (Fig. 1). Actin staining is also seen forming a circumferential ring associated with the adherens junctions at the apical surface of SCs. SCs are clearly differentiated from HCs with fluorescence microscopy 


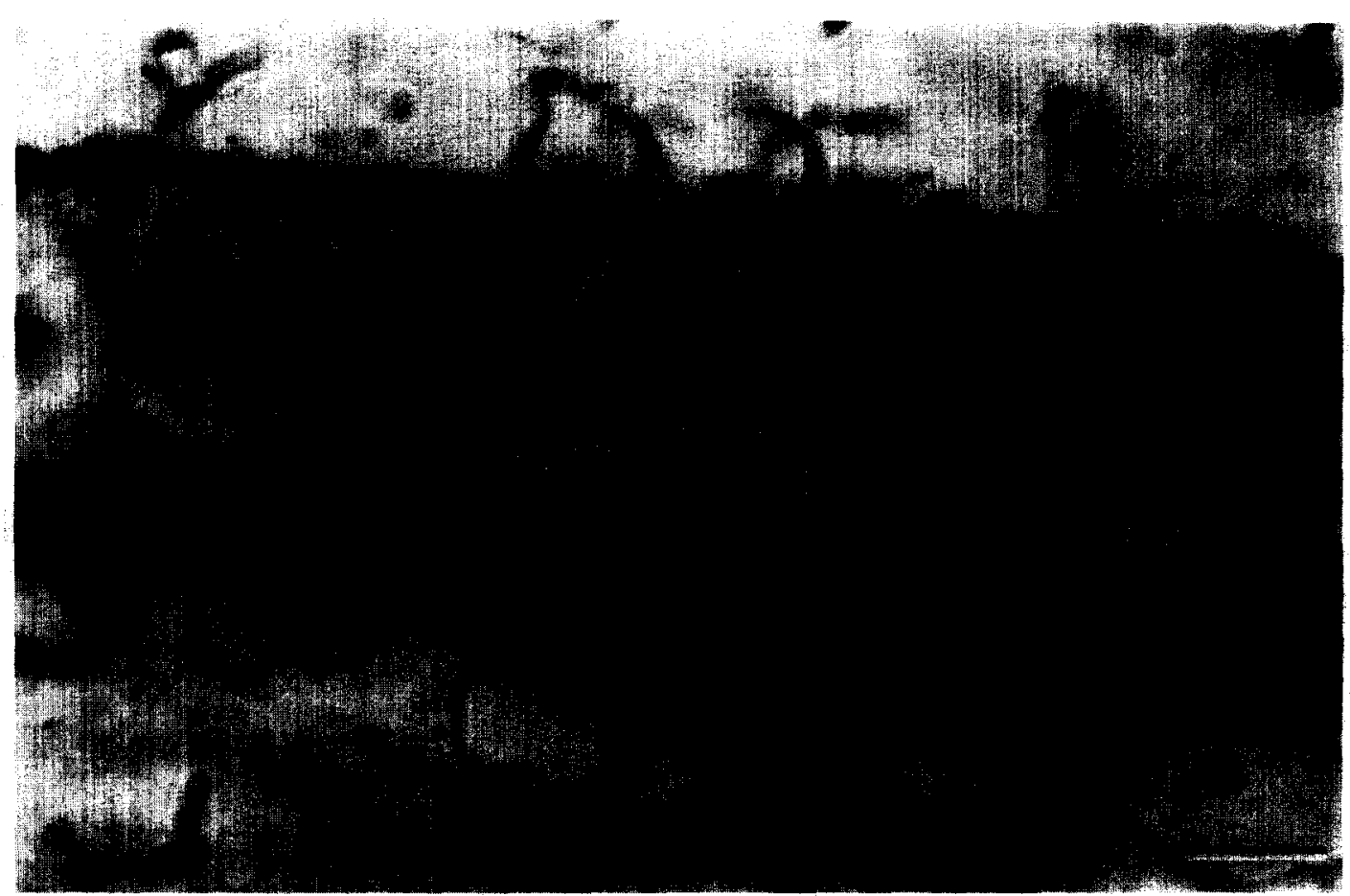

Fig. 4. A light micrograph of a plastic section from the normal crista ampullaris labeled for cytokeratins. Label is found in supporting cells only. The most intense label was at the apical domain of supporting cells (arrows). Type $I$ and II hair cells can be differentiated by the presence (type I) or lack of (type II) a nerve calyx. The nuclei of cells in this epithelium are segregated in a laminar fashion. with nuclei of supporting cells adjacent to the basement membrane. Bar, $10 \mu \mathrm{m}$.

when stained with actin, as they lack stereocilia and a cuticular plate. The luminal surface of SCs is polygonal in shape, ranging from four to six sides, as opposed to the $\mathrm{HC}$, whose luminal surface is circular. A narrow actin-free zone is seen between cells, demarcating the borders between adjacent cells. Together, the apical surfaces of HCs and SCs form a mosaic pattern.

There does not appear to be any regularity to the topographical architecture in the mosaic of $\mathrm{HCs}$ and SCs when viewed from above (Fig. 1). From 4-6 SCs surround each HC (Fig. 2). Occasionally, two HCs are seen abutting each other without an apparent intervening $\mathrm{SC}$.

HCs and SCs are also distinguishable based upon their cytokeratin staining characteristics (Meiteles and Raphael, 1994). Cytokeratin immunolabeling is confined to the SCs. There is a complete absence of cytoplasmic cytokeratin staining of HCs of the vestibular sensory epithelium. This is true in all five vestibular sensory organs. HCs appear as a signal void when the vestibular epithelium is immunolabeled with cytokeratin (Fig. 3).

Examination of LM sections of the vestibular sensory epithelium reveals a similar pattern of cytokeratin distribution, as observed by immunofluorescence. Cytokeratin staining is found only within SCs of the vestibular sensory epithelium (Fig. 4). Cytokeratin im- munostaining is seen throughout the $\mathrm{SC}$, with greatest intensity at the apical region.

The differential distribution of cytokeratin and actin in the auditory and vestibular epithelia (Raphael and Altschuler, 1991a; Meiteles and Raphael, 1994) is used here to study the cellular response to aminoglycoside toxicity. An aminoglycoside was directly applied into the round window and its effect on auditory and vestibular sensory epithelium investigated with immunofluorescence microscopy of whole tissue mounts. Cochlear surface preparations were examined and revealed complete HC loss. Scars were present throughout the auditory epithelium at 1 week post-treatment.

The unilateral application of aminoglycoside to one inner ear afforded us the ability to observe its effect clinically. Four hour after aminoglycoside application, the guinea pig developed nystagmus, of an ablative type, with the fast component beating away from the inner ear that was treated. The guinea pig also developed a head tilt toward the ear that was instilled with aminoglycoside and would tend to circle around in the direction toward the aminoglycoside treated ear. The animals tended to compensate within 24 to $48 \mathrm{~h}$ after the treatment, with complete resolution of the nystagmus and the tendency to circle around. The head tilt would occasionally persist for longer periods of time, up to 4 days after treatment. 
Control experiments were performed in which artificial perilymph was perfused into the inner ear instead of the aminoglycoside. This was performed in order to investigate the effect of our method for drug delivery upon the inner ear. To ensure adequate perfusion, the needle was inserted partially into the osseous spiral lamina. A concomitant mixing of endolymph and perilymph at the round window secondary to the perfusion occurred. In these control ears, an approximately $60 \%$ loss of inner $\mathrm{HCs}$ and $95 \%$ loss of outer HCs took place following the mechanical trauma in the cochlea (Fig. 5).

Interestingly, three different types of trauma may result in a similar pattern of scarring in the auditory epithelium. Scars were present in the organ of Corti similar to those seen in acoustical and chemical trauma. Specifically, the junctional complexes between supporting cells in scar region resembled these seen after other types of trauma (Raphael and Altschuler, $1991 \mathrm{a}, \mathrm{b})$. As in previous works using this method for labeling and analysis, the reticular lamina appeared intact at all times. This was verified with careful analysis of the reticular lamina with Nomarski optics. The vestibular epithelium from inner ears perfused with artificial perilymph were examined as whole tissue mounts and appeared normal and free of any scars.

Examination of the vestibular sensory epithelium treated with $10 \mathrm{mg}$ of streptomycin revealed an approximately 50 to $75 \%$ loss of the absolute number of HCs. Concentrations of $20 \mathrm{mg}$ of streptomycin perfused into the round window induced a complete loss of HCs. One day after treatment the vestibular epithelium had a normal appearance. Scar formation in the vestibular epithelium was seen 4 days after treatment with streptomycin. The scars were most often composed of expanded processes of three to five neighboring SCs (Figs. 6 and 7). The center of the scar, where the HC used to reside, was surrounded by a band of actin formed by all the SCs that participated in the scarring process [Fig. 6, demarcated line in (a) and (b)]. Within this domain, actin bridges divide the apical surface of the degenerated $\mathrm{HC}$ into different sized regions. The actin bands represent advancing processes of neighboring SCs filling the region of the degenerating $\mathrm{HC}$. An actin-free zone is seen in the apical surface of each advancing $\mathrm{SC}$, near the area of contact at the middle of the scar (Fig. 6C).

The distribution of cytokeratin was also altered during scar formation. Examination of the vestibular sensory epithelium double-labeled with fluorescent phalloidin (Fig. 8A) and cytokeratin-specific antibodies (Fig. $8 \mathrm{~B}$ ) revealed that the area once occupied by the degenerated $\mathrm{HC}$ is now replaced with the cytokeratin-rich processes of SCs. The remaining HCs that did not degenerate were devoid of cytokeratin staining (Fig. 8). Analysis with phase contrast and Nomarski optics
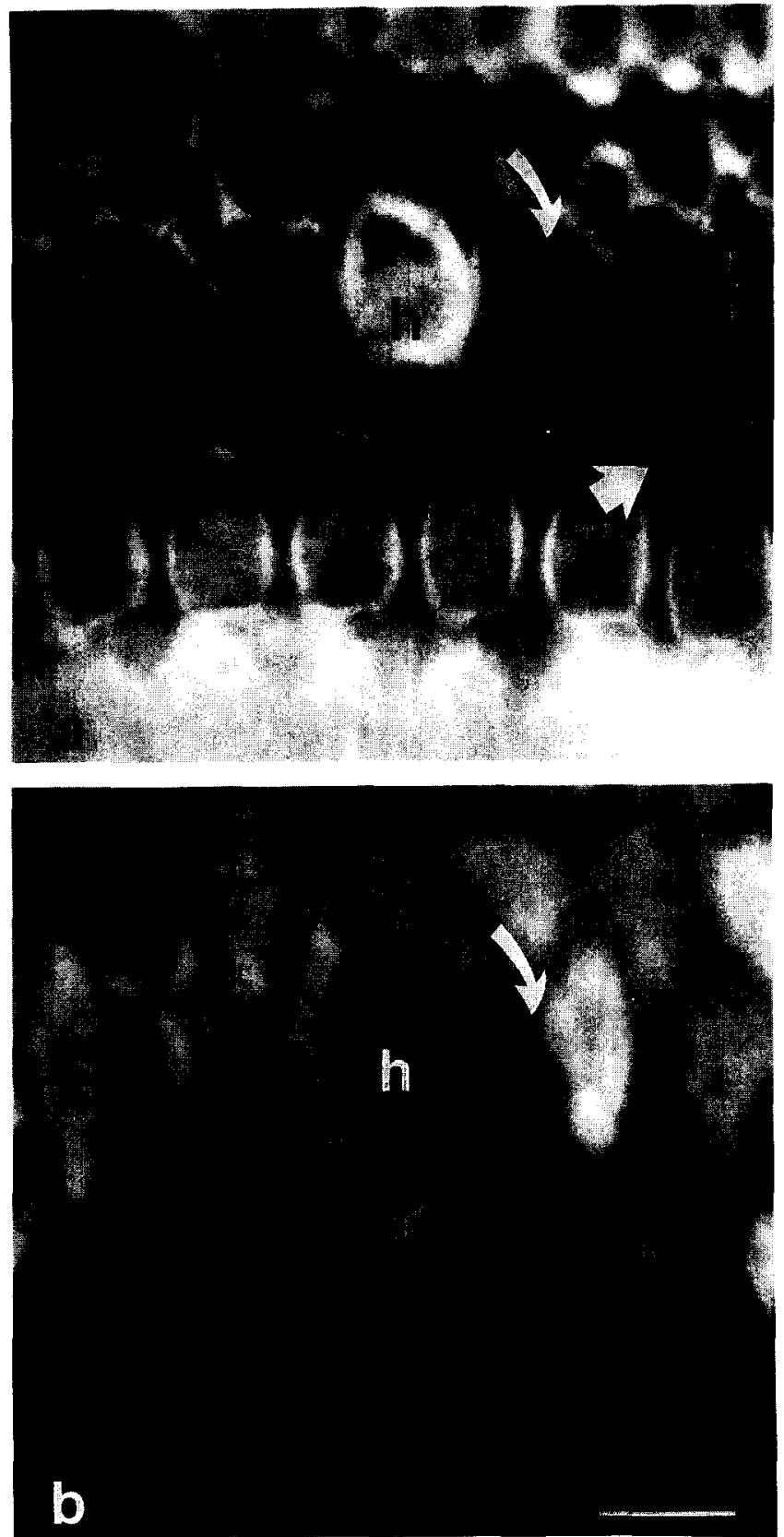

Fig. 5. A whole mount of the organ of Corti perfused with artificial perilymph, double-labeled with probes specific for actin (a) and cytokeratin (b). (a) Actin-specific label is seen in the cuticular plate of hair cells (h), in conjunction with adherens junctions between cells and in supporting cells. Scars can be seen in the second and third rows of outer hair cells. An actin band demareating the leading edge of the expanded supporting cell can be seen in the center of the degenerated hair cell (curved arrow). The phalangeal process of an outer pillar cell is also labeled for actin (straight arrow). (b) Cytokeratin-specific immunoreactivity in the same field as (a) reveals that the phalangeal processes of supporting cells are expanded, filling the space of degenerated hair cells (curved arrow). Remaining hair cells do not show cytokeratin-specific label (h). Bar. $10 \mu \mathrm{m}$

helped determine that the reticular lamina appeared confluent at all times.

LM cross sections of the vestibular sensory epithe- 

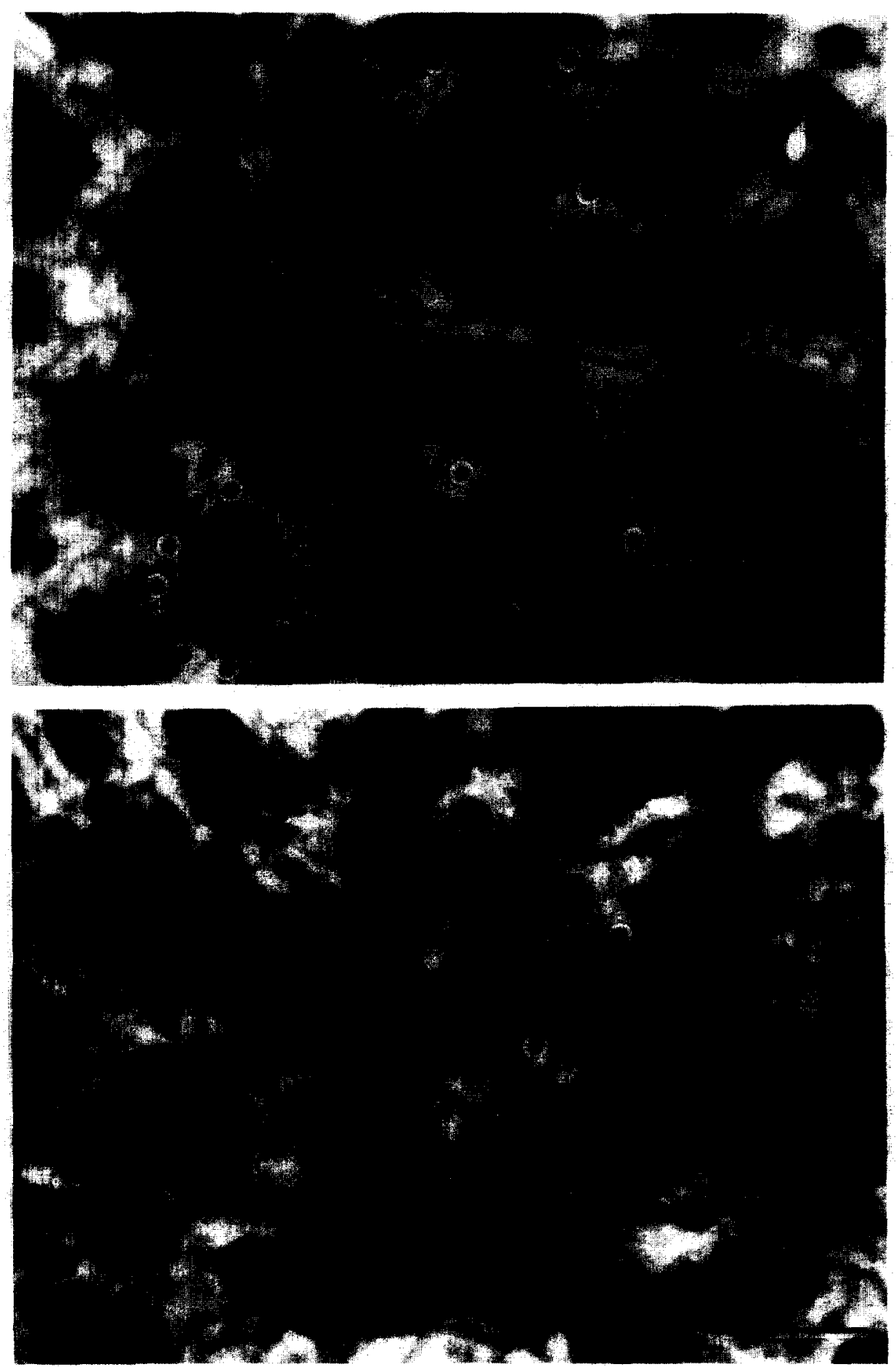

Fig. 6. Confocal laser micrograph of whole mount of the crista ampullaris, stained with phalloidin, 7 days after treatment with streptomycin. Scars are composed of the expanded processes of supporting cells that fill the gap left by degenerated hair cells. The area of the degenerated hair cell forms the center of the scar. Different patterns of scars are seen, depending on the number of supporting cells involved. (a) Three, four or five supporting cells participate in creating each scar. An example of each pattern is denoted by the black and white circles placed circumferentially around the region of degenerated hair cells. (b) The center of a scar (the area where the apical border of the hair cell had resided) is composed of the expanded processes of four supporting cells (demarcated by a circumferential ring of black and white circles). A thin, actin-free line is seen between the expanded processes of supporting cells, demarcating the borders between supporting cells. The center of the scar is surrounded by a band of intense actin staining. An apical border of a supporting cell involved in the scarring process is outlined with black squares. The supporting cell has maintained its polygonal shape and expanded to fill a portion of the region of the degenerated hair cell. Bar, $5 \mu \mathrm{m}$. (c) $\mathrm{A}$ schematic of scar formation in the vestibular epithelium. The black lines are cellular borders, the black spots are actin-free areas and the white areas represent actin-specific labeling. (A) One hair cell is in contact with six surrounding supporting cells in the normal vestibular epithelium. (B) The site of a degenerated hair cell is occupied by five expanded supporting cells. The apical membrane is confluent, whereas beneath the surface, an actin-free ring is found near the center of the scar. The ring is formed by an actin-free band in each expanded supporting cell. 
A

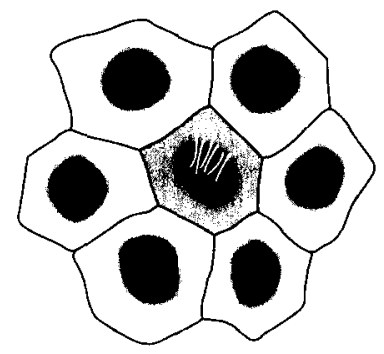

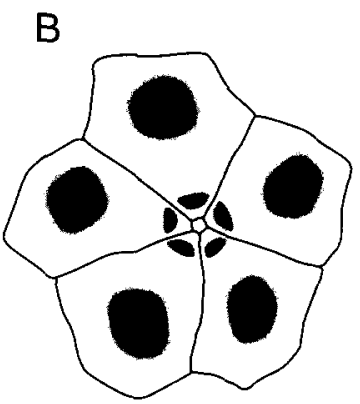

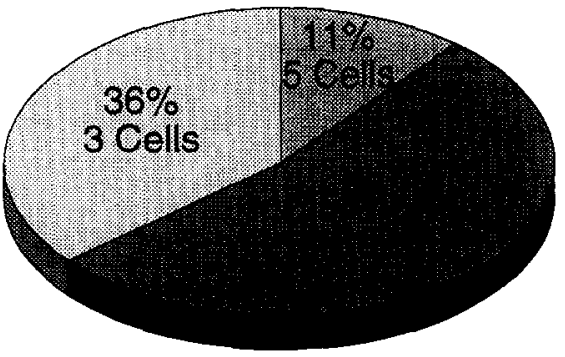

3 Cells $=36 \%$

4 Cells $=53 \%$

5 Cells $=11 \%$

Fig. 7. The number of supporting cells participating in each scar was counted $(N=100)$. Between three and five supporting cells form a scar for each individual hair cell. The majority of scars were formed by four supporting cells (compare with Fig. 2).

pand after aminoglycoside treatment and protrude into spaces they do not normally occupy.

Examination of the vestibular sensory epithelium from a guinea pig allowed to survive for 3 months revealed scars throughout the midportion of the crista ampullaris, where most HCs were missing (Fig. 10A). The apical surface of remaining SCs in scar regions were larger than usually seen in the untreated vestibular epithelium. The end portion of the crista ampullaris had a near normal complement of HCs. Some of the remaining $\mathrm{SCs}$ in this area were also seen to be larger
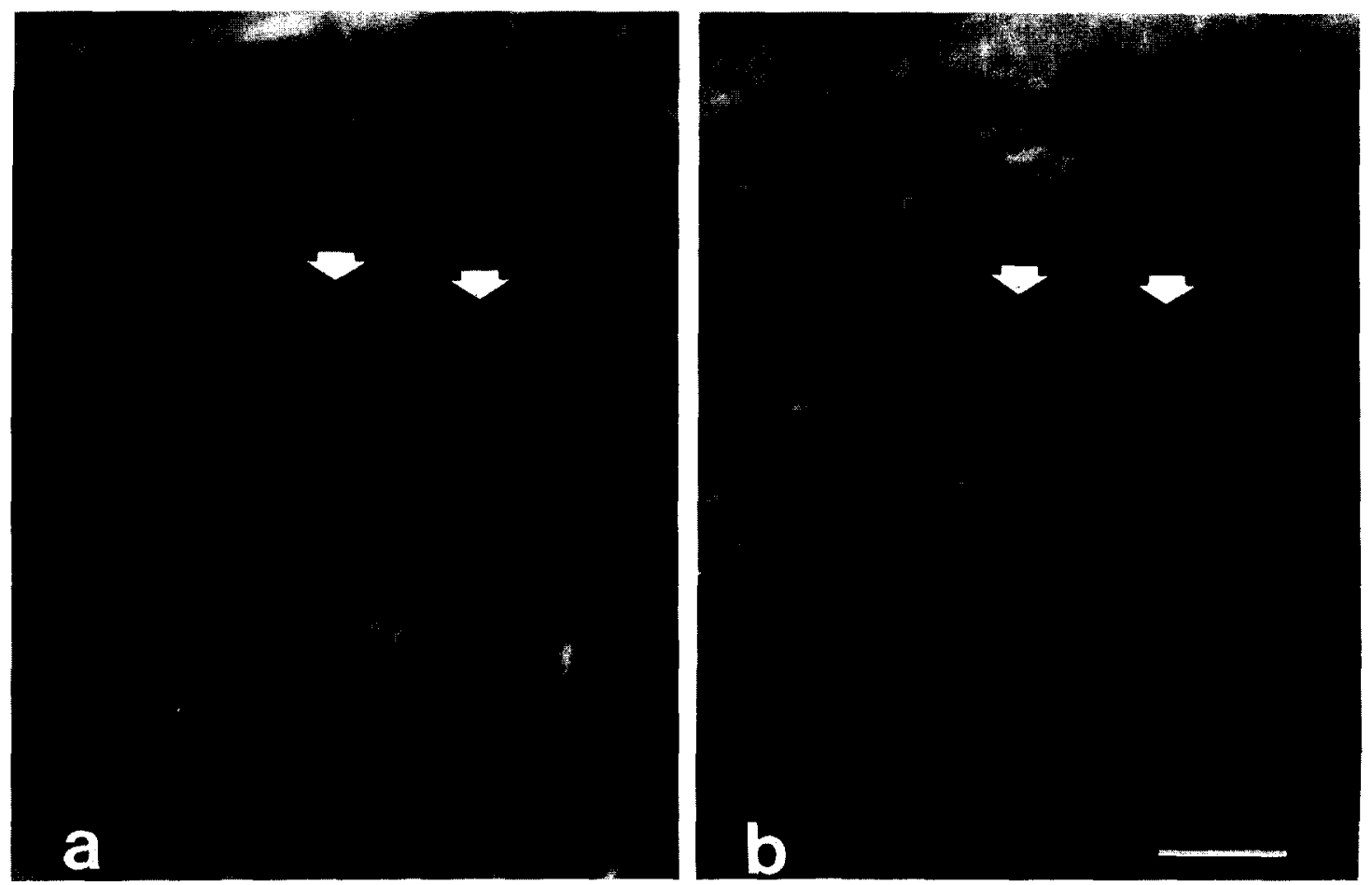

Fig. 8. Whole mount of the crista ampullaris double-labeled for actin and cytokeratins, 7 days after treatment with streptomycin. (a) Actin-specific labeling can be seen in the cuticular plate, stereocilia and forming a ring around the apical surface of hair cells and supporting cells. Scars are seen throughout the epithelium. Arrows point to hair cells that have not degenerated. (b) The same field as (a) showing cytokeratin staining in the epithelium. Cytokeratin-specific immunoreactivity is abundant wherever scars are present. whereas remaining hair cells (arrows) appear as dark circles lacking cytokeratin stain. Bar, $10 \mu \mathrm{m}$. 



Fig. 9. (a) Light micrograph of the crista ampullaris 4 days after streptomycin treatment, stained for cytokeratin. A continuous sheet of cytokeratins can be seen lining the luminal surface. Only one hair cell remains in the field (arrow), where cytokeratin-specific staining is absent. (b) Electron micrograph of the crista ampullaris 4 days after treatment with streptomycin. An intercellular junction between two supporting cells has formed in the horizontal plane parallel to the luminal surface (arrowheads). Such junctions are not seen in the normal cristat ampullaris. Bars, $10 \mu \mathrm{m}$ in (a) and $1 \mu \mathrm{m}$ in (b).

than usual (Fig. 10B). This probably represented a maturation process of SCs that have previously enlarged to form scars and occupy the space of degener- ated HCs. Immature HCs were not seen 3 months after aminoglycoside toxicity. It appeared that full recovery of the epithelium did not occur. 


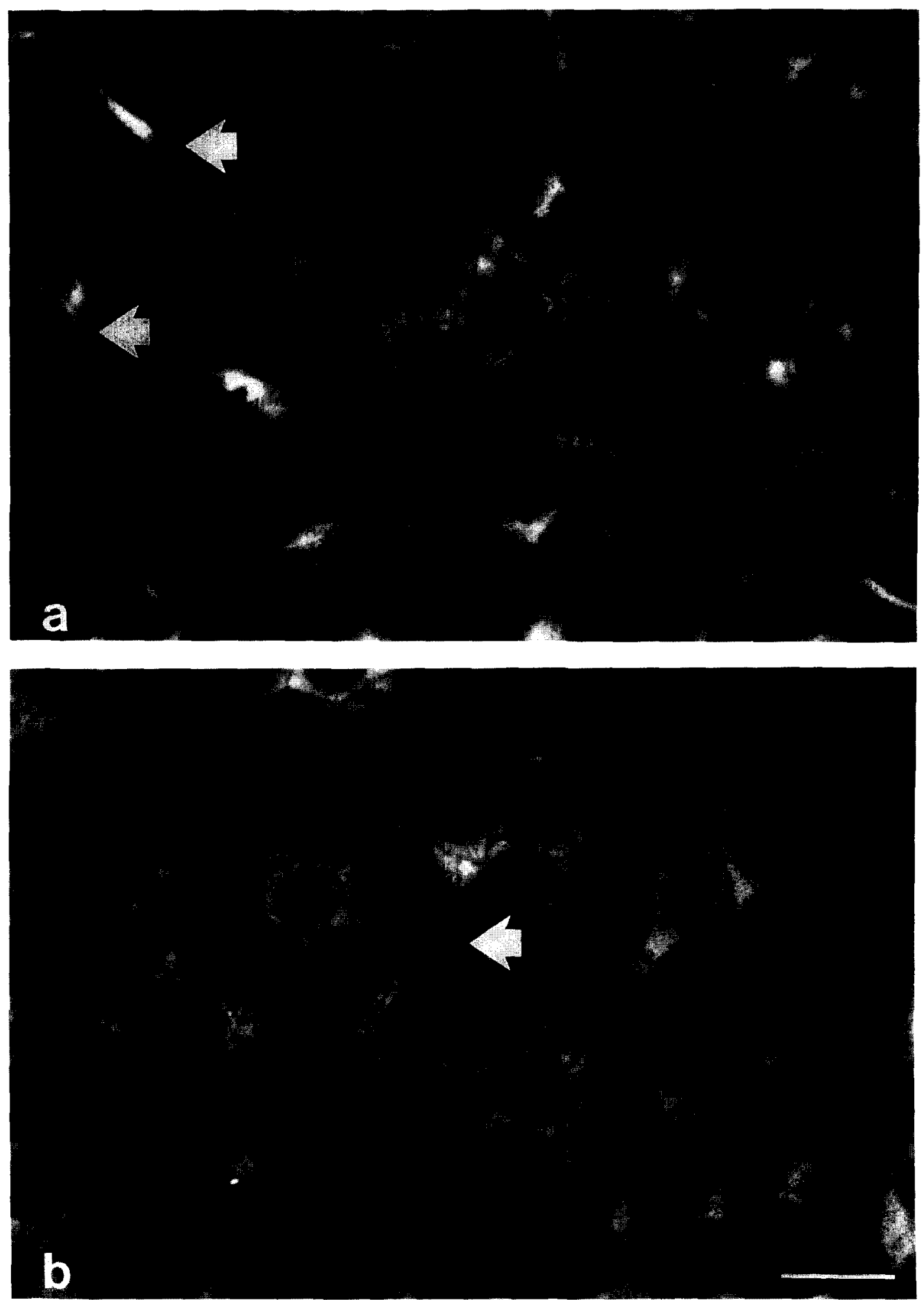

Fig. 10. Phalloidin-stained whole mount of the crista ampullaris 3 months after treatment with streptomycin, (a) In the end portion of the crista. near the planum semilunatum, a nearly normal complement of hair cells (arrows) is present. The apical surfaces of supporting cells have expanded. and many are larger than hair cells (compare to the normal epithelium. Fig. 1). (b) The midportion of the crista is almost completely devoid of hair cells. The apical surfaces of supporting cells are enlarged. filling the entire surface viewed. $A$ remaining hair cell is in the center of the field (arrow). Bar, $10 \mu \mathrm{m}$. 


\section{Discussion}

Examination of the vestibular sensory epithelium revealed a similar pattern of cytokeratin and actin staining compared to the auditory system (Raphael et al., 1987; Meiteles and Raphael, 1994). Cytoplasmic cytokeratin was not present within HCs of the inner ear sensory epithelium. Double labeling with actin and cytokeratin allowed us to co-localize these filaments and differentiate between HCs and SCs in both the normal and streptomycin-treated tissue.

Using these labeling methods, we determined that perfusion of artificial perilymph into the scala tympani and the concomitant mixing of endolymph and perilymph resulted in scar formation of the organ of Corti. The scarring process seen was similar to the scarring process documented after chemical- and noise-induced trauma of the mammalian organ of Corti (Raphael and Altschuler, 1991a,b). Using similar techniques, HC degeneration had not been seen on as large a scale as we documented (Hallen et al., 1974a,b; Nomura and Hara, 1986; Nomura et al., 1992). One possibility to explain the difference in the extent of the lesion is that the trauma produced by Nomura et al. probably did not result in the mixing of endolymph and perilymph.

The effect of streptomycin on the vestibular epithelium of the guinea pig has been previously investigated by phase-contrast microscopy of whole mounts (Lindeman, 1969). Collapse figures were identified in the vestibular sensory epithelium comparable to phalangeal scars in the organ of Corti. After a few weeks, the SCs, surrounding the degenerated $\mathrm{HCs}$, filled the gap formed by the lost $\mathrm{HC}$ and the collapse figure disappeared. The present study confirms and expands the findings by Lindeman. Scar formation was seen 4 days after the treatment with streptomycin. The scars were composed of expanded processes of three to five neighboring SCs, as determined by the presence of keratin-type intermediate filaments, SC-specific markers (Meiteles and Raphael, 1994). Regions where HCs degenerated and scars were present were filled with cytokeratin-rich processes of SCs.

Different types of scars were observed. On whole mounts of the surface epithelium stained with phalloidin, areas where HCs degenerated became filled with differing numbers of actin bridges. The apical surface of degenerated HCs was divided into differing numbers of spaces (three to five) of varying sizes. This may represent a continuum of stages of the scarring process. Initially, five neighboring SCs may enlarge to fill the gap formed by the degenerated HC. Ultimately, only three cells are necessary to form a permanent scar. If indeed this represented a continuum of stages, then one would expect to find five cells involved in the scarring process early on and only three cells involved at a later time. We believe this hypothesis is unlikely, because all stages of the scarring process were seen itt all time periods investigated.

The different types of scars seen may represent at differential pattern between scars of type I and type 11 $\mathrm{HCs}$. Once the $\mathrm{HC}$ has degenerated, we are unable to determine, from examination of the apical surface, whether the HC that degenerated was type I or type II. However, if the scars purely represented type I or type II HCs, then only two types of scars would be expected, and we are able to distinguish three different patterns.

We believe that the different patterns of scars seen represent the differing number of SCs surrounding each HC. In the normal mosaic of the vestibular sensory epithelium, between four and six SCs surround each HC. There is a tendency for five SCs to surround each HC. We document that three to five SCs form scars after the HC degenerates. There is a tendency for the scar pattern involving four SCs. There appears to be one cell less than the normal number of SCs surrounding each $\mathrm{HC}$ participating in the scarring process. The advantage that this scarring pattern offers the vestibular epithelium remains to be elucidated.

We document that the scarring process within the vestibular sensory epithelium is similar to the process described for the auditory sensory epithelium (Raphael and Altschuler, 1991a,b). In the organ of Corti, the normal outer $\mathrm{HC}$ is surrounded by the phalangeal processes of four SCs. During scar formation, only two SCs participate in the process. The two SCs in the same row as the degenerated $\mathrm{HC}$ expand and fill the space of the degenerated HC (Bohne, 1976; Hawkins and Johnson, 1981; Raphael and Altschuler, 1991a,b). It appears, in the vestibular sensory epithelium, that one cell less than the number of SCs surrounding the $\mathrm{HC}$ participates in scar formation. Similar to the organ of Corti, the reticular lamina appears to remain intact during the scarring process. A reorganization of the intermediate filament system and reticular lamina occurs during the scarring process. At the times investigated, a continuous reticular lamina was always seen, as observed by both immunofluorescence and LM. Early in the scarring process, actin bridges are seen in the area usually occupied by the HCs. We speculate that these actin bridges are adherens junctions that form between the advancing processes of SCs, helping to maintain an intact reticular lamina and probably preventing the mixing of fluids between different compartments of the inner ear.

Between 1 and 3 months after the initiation of the scarring process, the scars were composed of a sheet of SCs with enlarged apical surfaces. We noted that SC nuclei formed a bilayer in areas devoid of HCs. This may represent migration of nuclei during the scarring process. Whether the absolute number of SCs has increased or remains the same is unknown. We did not document any dividing cells within the vestibular ep- 
ithelium during the scarring process. Further experiments are planned to investigate whether SC divisions occur during the scarring process.

Recently, the guinea pig vestibular sensory epithelium was shown to have a limited regenerative capacity (Forge et al., 1993). HC loss around the striola of the utricle was induced by the daily injection of gentamicin for 10 days. One week after treatment, loss of $\mathrm{HCs}$ around the striola was evident, but 4 weeks after treatment, few immature HCs were seen within the striolar region. The stereocilia bundles for these immature HCs resembled the stereocilia of developing HCs. Regeneration of only $10 \%$ of the sensory epithelium occurred, and full recovery of the epithelium has not been documented. The ultimate fate of the immature HCs seen by Forge et al. is also unknown. We did not document $\mathrm{HC}$ regeneration in our study. If regeneration did occur, then it was incomplete or possibly reversed. Alternatively, it is possible that our treatment to induce $\mathrm{HC}$ loss was too harsh and prevented regeneration. Severe insults to the vestibular sensory epithelium resulting in large losses of HCs may also damage SCs. These cells were shown to divide after trauma in the lateral line organ (Balak et al., 1990) and in the basilar papilla and the vestibular epithelium of the chick (Raphael, 1992; Weisleder and Rubel, 1993, respectively).

We also documented that one cell less than the number of SCs surrounding a $\mathrm{HC}$ participates in the scarring process. Perhaps the one cell that does not participate in the scarring process is destined to be the pluripotential cell that regenerates a $\mathrm{HC}$. If the $\mathrm{HC}$ loss is too severe and all SCs are involved in the scarring process, then the ability to regenerate $\mathrm{HCs}$ may be forfeited. Further experiments are needed to determine if the above scenario is true.

In conclusion, we have described the reparative process after $\mathrm{HC}$ degeneration in the vestibular epithelium due to streptomycin toxicity. Examination of the vestibular sensory epithelium reveals a similar pattern of cytokeratin and actin staining compared to the auditory system. Cytoplasmic cytokeratin is not present within HCs of the vestibular epithelium. Double labeling with actin and cytokeratin allowed us to co-localize these filaments and differentiate between HCs and SCs in both the normal and streptomycin-treated tissue. Scar formation involves the expansion and protrusion of SCs into the domain of degenerating HCs. Differing numbers of SCs are involved in the vestibular reparative process, in contrast with the auditory epithelium, where, in most cases, only two cells are involved. Three to five cells partake in the reparative process. Intercellular junctions are maintained between SCs participating in scar formation. The reticular lamina appears intact at all times, probably preventing mixing of fluids between different inner ear compartments. We did not document any evidence for $\mathrm{HC}$ regeneration, perhaps due to the severe HC loss inflicted by our trauma.

\section{Acknowledgments}

We would like to thank Peter A. Finger for excellent technical assistance and Christina A. Triezenberg for valuable help during preparation of the manuscript.

\section{References}

Balak, K.J., Corwin, J.T. and Jones, J.E. (1990) Regenerated hair cells can originate from supporting cell progeny: Evidence from phototoxicity and laser ablation experiments in the lateral line system. J. Neurosci. 10, 2502-2512.

Bohne, B.A. (1976) Healing of the noise damaged inner ear. In: Hirsh, S.K., Eldredge, D.H., Hirsh I.J. and Silverman, S.R. (Eds.), Hearing and Davis: Essays Honoring Hallowell Davis, Washington University Press, St. Louis, pp. 85-96.

Duvall, A.J. and Wersall, J. (1963) Site of action of streptomycin upon inner ear sensory cells. Acta Otolaryngol. 57, 581-598.

Fee, W.E., Jr. (1980) Aminoglycoside ototoxicity in the human. Laryngoscope 90 (Suppl. 24), 1- 19.

Forge, A., Li, L., Corwin, J.T. and Nevill, G. (1993) Ultrastructural evidence for hair cell regeneration in the mammalian inner ear. Science 259, 1616-1619.

Fowler, E., Jr. (1948) Streptomycin treatment of vertigo. Trans. Am. Acad. Ophthalmol. Otolaryngol. 52, 293-301.

Hallen, O., McPherson, D.L., Axelsson, A. and Miller, J.M. (1974a) Long-term morphological and electrophysiological effects of small mechanical lesions in the guinea pig cochlea. Acta Otolaryngol. 78. 304-320.

Hallen, O.. McPherson, D.L., Axelsson. A. and Miller, J.M. (1974b) Acute electrophysiological and morphological effects of mechanical trauma in the guinea pig cochlea. Acta Otolaryngol. 78 . $162-172$.

Hawkins, J.E., Jr. (1976) Drug Ototoxicity. In: W.D. Keidel and W.D. Neff (Eds.) Handbook of Sensory Physiology, Springer Verlag, Berlin, Vol. V3, p. 707

Hawkins, J.E., Jr. and Johnson, L.G. (1981) Histopathology of cochlear and vestibular ototoxicity in laboratory animals. In: Lerner, S.A., Matz. J.G. and Hawkins, J.E., Jr. (Eds.), Aminoglycoside Ototoxicity, Little, Brown \& Co.. Boston, pp. 175-195.

Hinshaw, H. and Feldman, W. (1945) Streptomycin in treatment of clinical tuberculosis: a preliminary report. Proc. Staff Meet. Mayo Clinic, 20, 313-318.

Langman, A., Kemink, J.L. and Telian, S.A. (1992) Titration streptomycin therapy. Operative Tech. Otolaryngol. Head Neck Surg. 2. $45-46$.

Lindeman, H.H. (1969) Regional differences in sensitivity of the vestibular sensory epithelia to ototoxic antibiotics. Acta Otolaryngol. $67,177-189$.

McGee, T.M. and Oiszewski, J. (1962) Streptomycin sulfate and dihydrostreptomycin toxicity. Arch. Otolaryngol. 75, 295-311.

Meiteles, L.Z. and Raphael. Y. (1994) Distribution of cytokeratin in the vestibular epithelium of the guinea pig. Ann. Oto. Rhinol. Laryngol. 103, 149-155.

Nedzelski, J.M., Chiong, C.M., Fradet, G., Schessel, D.A.. Bryce, G.E. and Pfleiderer, A.G. (1993) Intratympanic gentamycin instillation as treatment of unilateral Meniere's disease: update of an ongoing study. Am. J. Otol. 14, 278-282.

Nomura, Y. and Hara, M. (1986) Experimental perilymphatic fistula. Am. J. Otolaryngol. 7, 267-275. 
Nomura, Y., Hara, M., Young, Y.H. and Okuno, T. (1992) Inner ear morphology of experimental perilymphatic fistula. Am. J. Otol. $13,32-37$.

Raphael, Y. (1992) Evidence for supporting cell mitosis in response to acoustic trauma in the avian inner ear. J. Neurocytol. 21, 663-671.

Raphael, Y., Marshak, G., Barash, A. and Geiger, B. (1987) Modulittion of intermediate filament expression in the developing cochlear epithelium. Differentiation 35, 151-162.

Raphael, Y. and Altschuler, R.A. (1991a) Reorganization of cytoskeletal and junctional proteins during cochlear hair cell degeneration. Cell Motil. Cytoskeleton 18, 215-227.
Raphael Y. and Altschuler, R.A. (1991b) Scar formation after druginduced cochlear insult. Hear. Res. 51, 173-184.

Schuknecht, H.F. (1974) Pathology of the Ear, Harvard University Press, Cambridge, MA, and London, pp. 273-278.

Warchol, M.E., Lambert, P.R., Goldstein, B.J.. Forge, A. and Corwin, J.T. (1993) Regenerative proliferation in inner ear sensory epithelia from adult guinea pigs and humans. Science 259,1619 1622.

Weisleder, P. and Rubel. E.W (1993) Hair cell regeneration after streptomycin toxicity in the avian vestibular epithelium. J. Comp. Neurol. 33, 97-110. 